\title{
Development of a Porous Scaffold-Manufacturing Method by Blending Silk Fibroin and Agarose Polymer Solutions
}

\author{
Seung-Won Park*, HaeYong Kweon, Tae-Won Goo, Seong Ryul Kim, You-Young Jo, and Gwang-Ho Choi \\ Department of Agricultural Biology, National Academy of Agricultural Science, Rural Development Association, Suwon 441- \\ 100, South Korea
}

(Received 15 May 2012; Accepted 4 September 2012)

\begin{abstract}
Low-melting-temperature agarose gel solution, as a novel porogen was combined with a silk fibroin solution to generate interconnected porous networks. The porosity of the resultant silk fibroin-agarose scaffolds was greater than that of the scaffolds generated with agarose and deionized water. The porosities of silk fibroin scaffolds containing agarose gel at $0.5 \%, 1.0 \%$, $1.5 \%, 2.0 \%$ [w/v] were $110.9 \%, 111.7 \%, 120.9 \%$, and $123.0 \%$, respectively. Lastly, the internal space generated in scaffolds after dissolution of the agarose gel provides a good environment for cell growth and movement within the scaffold.
\end{abstract}

Key words: Silk fibroin, Agarose hydrogel solution, Scaffold, Tissue engineering

\section{Introduction}

The microenvironment of three dimension (3D) scaffolds is important for cell attachment, proliferation, and penetration within the scaffold. Consequently, much of tissue engineering research has focused on the utilization of porous sponge structures, owing to their microenvironment and adaptability to various complicated morphologies (Makaya et al., 2009). Sofia et al. (2001) first described the microstructure of a silk porous scaffold. Using salt-leaching methods, the authors developed scaffolds varying in porosity by altering silk solution con-

*To whom the correspondence addressed

Sericultural \& Apicultural Materials Division, National Academy of Agricultural Science, RDA, Suwon 441-100, Republic of Korea.

Tel: +82-31-290-8493; Fax +82-31-290-8503;

E-mail: microsw@korea.kr

http://dx.doi.org/10.7852/ijie.2012.25.1.075 centrations and salt particle sizes (Kim et al., 2005). Although the procedure was successful in generating larger size porous silk scaffold by using salt particles of $1,000 \mu \mathrm{m}$, it was inadequate for use with salt particles less than $500 \mu \mathrm{m}$ (Makaya et al., 2009).

Furthermore, scaffolds irregular internal pore sizes cause technical difficulties in the establishment of accurate external structures and, subsequently, cell culture systems. Recent studies have explored scaffold-manufacturing, techniques in an attempt to maintain acceptable microenvironment properties. Sarazin et al. $(2003$; 2004) developed a novel method for the generation of ultra-porous scaffolds by melt blending 2 or 4 immiscible polymers (Virgilio et al., 2010). Roy et al. (2006) deposited polymer/ protein layers onto scaffold surfaces to increase the porosity of the materials. Virgilio et al. $(2005 ; 2009 ; 2009$; 2010) and Horiuchi et al. (1997) reported partial wetting in a significant number of scaffolds generated by ternary immiscible polymers blends.

In the present study, by melt blending of solutions derived from silk fibroin and agarose polymers, we developed a novel manufacturing method for fully interconnected porous scaffolds. Low-melting-temperature agarose gel solution-, used as a novel porogen, was combined with silk fibroin solution to generate the ultra-porous 3D structure of scaffold. While agarose gel dissolves in warm water, silk fibroin scaffold does not. As a result, in silk fibroin-agarose scaffolds generated using a melt processing technique, the large internal spaces within the scaffolds contain dissolved agarose. Porosity and interconnected porous networks of silk sponge scaffolds can be regulated by altering the ratio of silk fibroin solution to agarose gel solution. Lastly, the internal space generated in scaffolds after dissolution of -agarose gel provides a good environment for cell growth and movement within the scaffold. Since silk and agarose are derived from natural products, they are biocompatible with the human body. Silk fibroin-agarose scaffolds, therefore, are non-toxic, 
as they will not cause a foreign body immune response that other synthetic polymers can induce.

\section{Materials and Methods}

\begin{abstract}
Materials
Low-melting-temperature agarose, SeaPlaque ${ }^{\circledR}$ Agarose, was provided by Lonza Corp. (Rockland, ME). The agarose melts with distilled water under $65^{\circ} \mathrm{C}$ and had a gelling temperature from $26^{\circ} \mathrm{C}$ to $30^{\circ} \mathrm{C}$. Cocoons of Bombyx mori silkworm were obtained from National Academy of Agricultural Science (Suwon, Korea). Silk was boiled off for 30 min in an aqueous solution of $0.02 \mathrm{M} \mathrm{Na}_{2} \mathrm{CO}_{3}$ and rinsed thoroughly with water to produce silk fibroin proteins.
\end{abstract}

\section{Preparation of silk fibroin solution}

Purified silk fibroin was dissolved in $\mathrm{CaCl}_{2} / \mathrm{EtOH} /$ water solution for $5 \mathrm{~h}$. The solution was dialyzed in water for $72 \mathrm{~h}$ using cellulose membrane (molecular cutoff = $12,000 \sim 14,000)$ at room temperature.

\section{Aqueous-derived agarose polymer preparation}

To prepare a $100 \mathrm{ml}$ stock of $3.0 \%$ agarose stock solution, add $3.0 \mathrm{~g}$ agarose to $100 \mathrm{ml}$ sterile deionized water in a $250 \mathrm{ml}$ Pyrex media bottle, cap loosely and completely dissolved in the microwave. Mix well as soon as the microwave cycle has finished (being careful to avoid splashing of the hot liquid) and keep with $200 \mathrm{ml}$ of deionized water in the water bath at $50^{\circ} \mathrm{C}$. Before silk fibroin-agarose polymer solution blend, 3.0\% agarose stock solution was diluted with deionized water at weight ratios of $0.5,1.0,1.5$, and $2.0 \%[\mathrm{w} / \mathrm{v}]$.

\section{Preparation of silk fibroin-agarose polymer solution blend scaffold}

Aqueous-derived silk fibroin solution (10\%) was mixed with sterile deionized water or different concentrations of agarose solution at ratio of $1: 1[\mathrm{v} / \mathrm{v}]$. To a casting the scaffolds, the mixed solutions were poured into a 24 -well tissue culture plate $(2 \mathrm{ml} /$ well $)$ and allow the plates to cool and keep at $4^{\circ} \mathrm{C}$ to solidification of agarose. The plates dried at $-80^{\circ} \mathrm{C}$ in a freeze-drying oven for $72 \mathrm{~h}$. The resultant scaffolds were incubated with absolute ethanol for $1 \mathrm{~h}$ in order to induce insolubility of silk fibroin against water. To remove all of residual agarose molecules, a used agarose molecules could be melts with water under $65^{\circ} \mathrm{C}$. In the immersing step, the scaffolds were immersed in the $50 \mathrm{ml}$ cap tube filled with deionized water and incubated to water bath at $60^{\circ} \mathrm{C}$ for 2 days. The internal deionized water of $50 \mathrm{ml}$ cap tube was changed with new water (total 6 times for 2 days).

\section{Scanning electron microscopy (SEM)}

The morphology of the scaffold had been analyzed by a scanning electronic microscope (Hitachi S-3500, Japan). SEM was done at Regional Research Center, Myongji University, South Korea.

\section{Swelling test}

To determine the water absorption efficiency of scaffolds, swelling studies were performed according to the procedures in the previous study (Mathew et al., 2010). Briefly, scaffolds were immersed in Hexane and temperature of $37^{\circ} \mathrm{C}$. The dry weight of the scaffold was noted (Wo). Scaffolds were placed in Hexane and after a predetermined time $(24 \mathrm{~h})$ scaffolds were taken out, surface adsorbed water was removed by filter paper and wet weight was recorded $(\mathrm{Ww})$. The ratio of swelling was determined using the following formula: Swelling ratio = (Ww - Wo)/Wo.

\section{Results and Discussion}

\section{Development of agarose-silk fibroin scaffolds}

For the generation of ultra-porous scaffolds, an aqueousderived silk fibroin solution was mixed with various concentrations of an agarose hydrogel solution, the porogen, at a ratio of $1: 1(\mathrm{v} / \mathrm{v})$. As shown in the schematic diagram in Fig. 1, the 2 polymer blends were dissolved in an aqueous solution. Water, therefore, was present between silk fibroin and agarose molecules. Because upon water evaporation, the aqueous-derived mixed solution could parti-

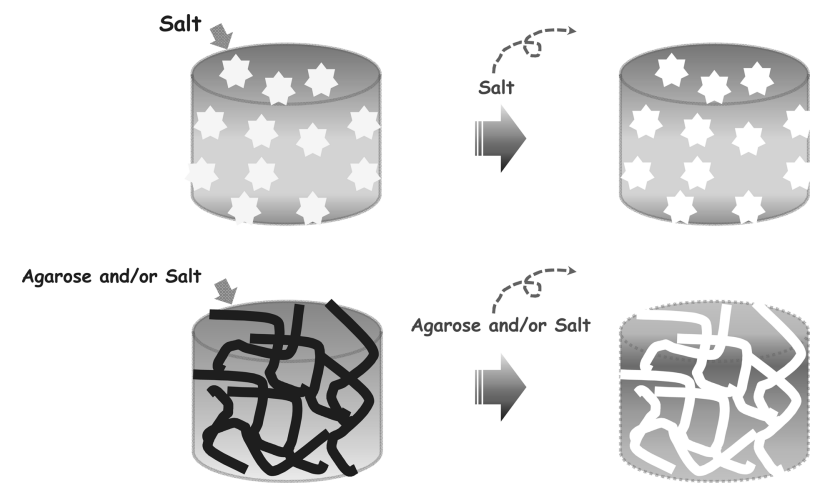

Fig. 1. A schematic diagram representing the development of silk fibroin-agarose scaffold. Silk fibroin and agarose polymer blends were dissolved in an aqueous solution. Water, therefore, was present between silk fibroin and agarose molecules. Fully interconnected porous scaffolds were prepared using the 2 immiscible polymers and a blending technique. Silk fibroin solution derived from $B$. mori cocoons was mixed with different concentrations of agarose hydrogel solution at a ratio of $1: 1$ $[\mathrm{v} / \mathrm{v}]$. 


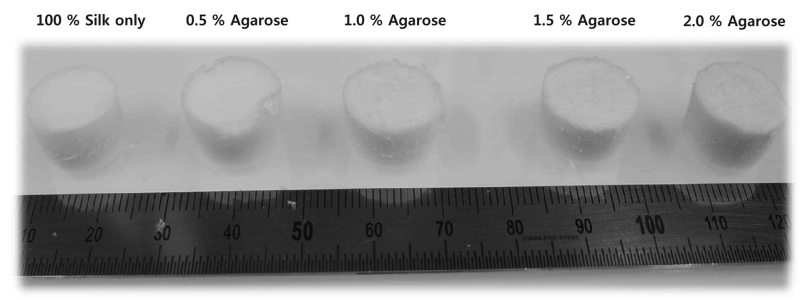

Fig. 2. Morphology of silk fibroin-agarose scaffolds. A 24-well tissue culture plate was used to cast scaffolds.

tion, we performed a freeze-drying step during scaffold preparation. We assumed, however, that agarose molecules would remain attached to the silk fibroin scaffolds. To remove the residual agarose molecules, after freezingdrying treatment, we immersed in warm deionized water for $48 \mathrm{~h}$. The portion of internal space in the resultant scaffolds was greater than that before the immersion step, and the internal space could be regulated by altering the ratio of silk solution to agarose solution.

\section{Morphological analysis of scaffolds}

Fully interconnected porous scaffolds were constructed in 24-well tissue culture plates by using 2 different types of polymers and a blending technique. Briefly, a silk fibroin solution derived from $B$. mori cocoons was mixed with varying concentrations of agarose hydrogel solution, the porogen, at a ratio of 1:1 [v/v] (Fig. 1). Prior to agarose leaching, the scaffold was in the shape of a cylinder, with a diameter of over $1.2 \mathrm{~cm}$ and a height of approximately $1.0 \mathrm{~cm}$ (Fig. 2). There was no change in scaffold size after freeze-drying (Fig. 2). To determine if the presence of water between silk fibroin and agarose molecules could influence the structure of scaffolds, we created spongetype scaffolds. A agarose leaching and freeze-drying of the scaffolds demonstrated that the agarose gel act as a porogen, creating an internal ultra-porous structure within the scaffold. The sponge like scaffolds absorbed water, their shape did not change (Fig. 3 A and B). Micro-structure characterization of both the silk fibroin-agarose and pure scaffolds was performed with scanning electron microscopy (SEM). SEM image of the scaffold internal microenvironment revealed that the pores in agarose hydrogel mixed silk scaffold were much larger than the pores of pure scaffold (Fig. 3C).

Morphologically, we observed the porous structure of silk fibroin-agarose blended scaffolds. The volume of the two polymer blended scaffolds were measured and compared to the pure silk scaffolds. The described silk fibroinagarose scaffold development technique is novel scaffoldmanufacturing procedure with 2 benefits: (1) economically, it is useful because of the inexpensive nature of the

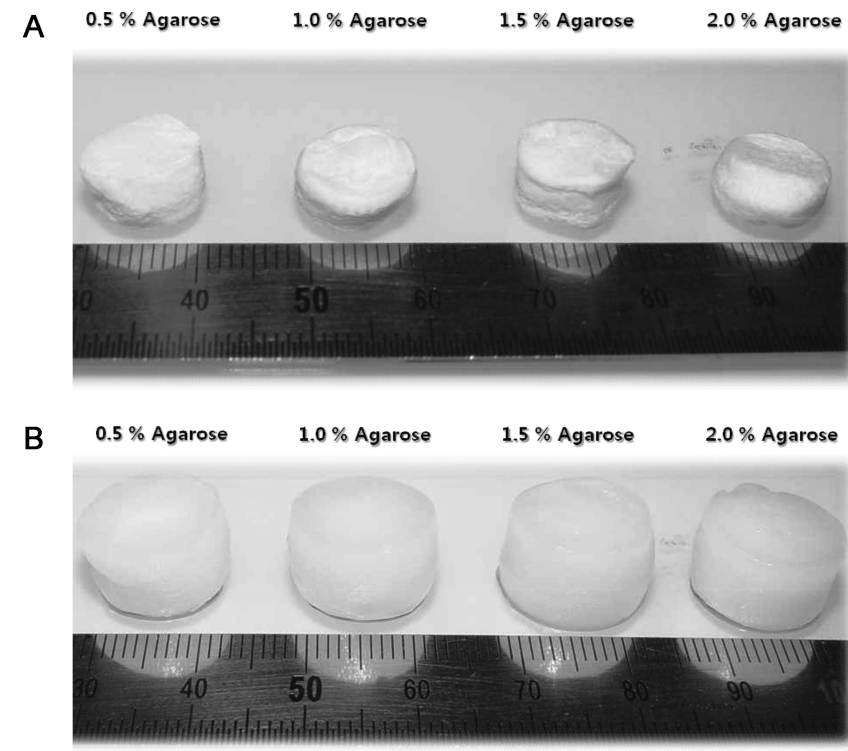

C
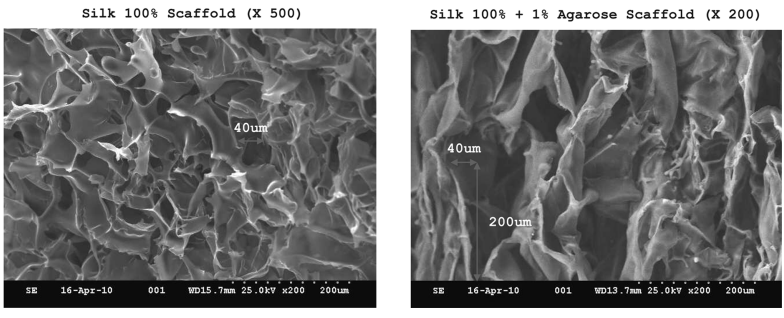

Fig. 3. Micro-structure characterization of silk fibroin-agarose scaffolds. A. Scaffold structure after agarose leaching and freeze-drying. B. Scaffold structure after the addition of water. C. Scanning electron microscopy images of scaffold internal microenvironments.

materials used, and (2) immunologically, because warm water substitutes the need for other, harmful solvents in scaffold generation, the resultant scaffold will not stimulate an immune reaction.

\section{Internal capacity analysis of scaffolds}

Liquid intrusion analysis was used to determine the porosity of scaffolds, containing various concentrations of hydrogel solution (Soliman et al., 2011). Aqueous-derived silk fibroin solution was mixed with deionized water or agarose hydrogel solution, the porogen, at a ratio of 1:1 $[\mathrm{v} / \mathrm{v}]$. The porosity was greater in the silk fibroin solution mixed scaffold than in the deionized water mixed scaffold (Fig. 4A). The porosity of the silk fibroin-agarose scaffolds increased with increasing hydrogel solution concentration [w/v] (Fig. 4B). The scaffolds were arbitrarily assigned to 4 groups differing in agarose hydrogel solution concentration $[\mathrm{w} / \mathrm{v}]$. The porosity of hydrogel silk scaffolds was greater than that of silk pure scaffold. Based on normalization to pure silk scaffold porosity, the poros- 
A
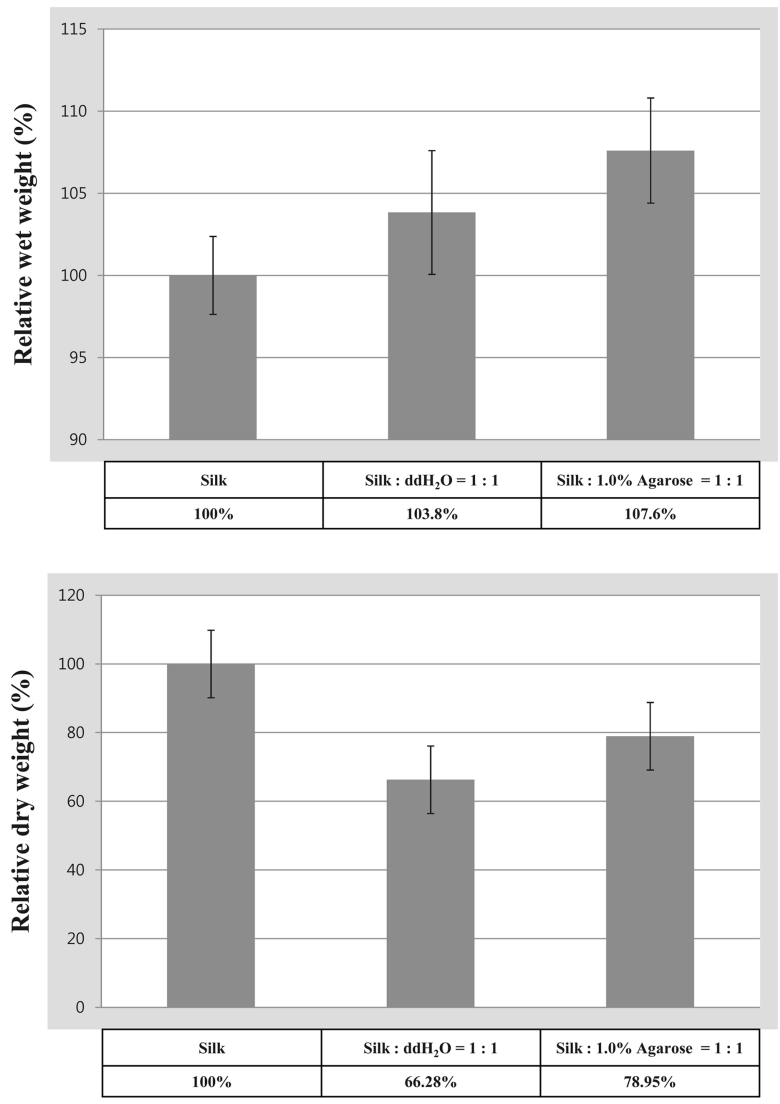

B

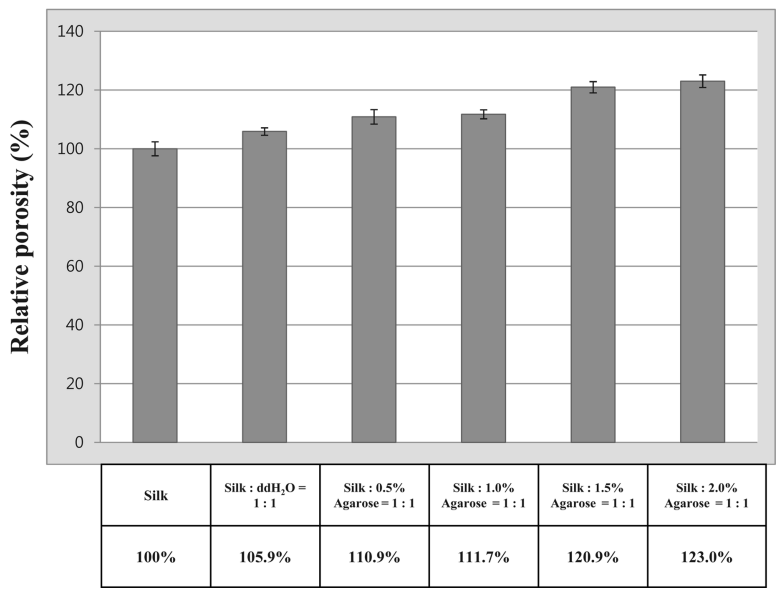

Fig. 4. Porosity analysis of silk fibroin-agarose scaffolds. A. The porosity shows to higher increase with the silk fibroin solution mixed scaffold than deionized water mixed scaffold. B. The scaffolds were arbitrarily assigned into four groups based on the concentration of agarose hydrogel solution $[\mathrm{w} / \mathrm{v}]$. Compared with pure silk scaffold, porosity of hydrogel mixed scaffolds were shown higher.

ities of scaffolds containing agarose gel were $110.9 \%$ on $0.5 \%[\mathrm{w} / \mathrm{v}]$ agarose, $111.7 \%$ on $1.0 \%[\mathrm{w} / \mathrm{v}]$ agarose, $120.9 \%$ on $1.5 \%[\mathrm{w} / \mathrm{v}]$ agarose, and $123.0 \%$ on $2.0 \%$ [w/ v] agarose. Therefore, $1.5 \%$ agarose was sufficient to generate ultra-porous silk scaffold.

The microenvironment of 3D scaffolds is important for cell attachment, proliferation, and penetration within the scaffold. Previously, regulation of pore size within scaffolds was induced using salt leaching methods (Makaya et al., 2009). Although the regulation of porous structure by using salt particles of varying size has its advantages, it does not overcome issues pertaining to the integration of fully interconnected porous networks with symmetric channels into scaffolds, which are required to guide the migration of cells into internal pores. Recently, alternatives to salt were used as porogens in the development of scaffolds. Sarazin, Favis (2003) and Virgilio et al. (2010) developed a new method for generating porous Poly-LLactide Acid (PLLA) scaffold by blending immiscible polymers. The resultant porous scaffold structure had a fully interconnected porosity and a highly controlled morphology (Virgilio et al., 2010). Although this method can be successfully applied to the development of porous 3D scaffolds, we argue that it is necessary to reduce chemical scaffold manufacturing, including the use of chemical solvents, as a protective measure against a immune response. Our novel silk fibroin-agarose scaffold-manufacturing method uses only biomaterials derived from natural products, thus eliminating the probability of stimulating an immune reaction. Futhermore, additional steps in scaffold development are unnecessary, because the agarose-leaching treatment essentially replaces salt leaching.

Lastly, the observed increase in interconnectivity between pores in our silk fibroin-agarose scaffolds will like aide in guiding the migration of cells into internal pores. In summary, the main benefits of our novel scaffold-manufacturing method are as follows : (1) the 2 polymers used are biocompatibile with the human body, (2) the technique for the removal of agarose molecules is relatively simple, (3) the interconnected internal space within scaffolds is uniform and can be regulated by altering the ratio of silk solution to agarose solution, and (4) the combination of salt and agarose hydrogel solution can aid in the development of highly porous and ultra-interconnected scaffold structures.

\section{Acknowledgement}

This research was supported by Bio-industry Technology Development Program, Ministry for Food, Agriculture, Forestry and Fisheries, Republic of Korea (grant no. 311059-4) and partially supported by the Rural Development Administration, Republic of Korea (grant no. PJ008475022012). 


\section{References}

Horiuchi S, Matchariyakul N, Yase K, Kitano T (1997) Morphology development through an interfacial reaction in ternary immiscible polymer blends. Macromolecules 30, 3664e70.

Kim UJ, Park J, Kim HJ, Wada M, Kaplan DL (2005) Threedimensional aqueous-derived biomaterial scaffolds from silk fibroin. Biomaterials 26, 2775-2785.

Makaya K, Terada S, Ohgo K, Asakura T (2009) Comparative study of silk fibroin porous scaffolds derived from salt/water and sucrose/hexafluoroisopropanol in cartilage formation. $\mathrm{J}$ Biosci Bioeng 108, 68-75.

Mathew P, Nitya G, Selvamurugan N, Nair SV, Furuike T, Tamura H, Jayakumur R (2010) Preparation and characterization of chitosan-gelatin/nanohydroxyapatite composite scaffolds for tissue engineering applications. Carbohydrate Polymers 80, 687-694.

Roy X, Sarazin P, Favis BD (2006) Ultraporous nanosheath materials by layer-bylayer deposition onto co-continuous polymer-blend templates. Adv Mat 18, 1015e9.

Sarazin P, Favis BD (2003) Morphology control in co-continuous poly(L-lactide)/polystyrene blends: a route towards highly structured and interconnected porosity in poly(L-lactide) materials. Biomacromolecules 4, 1669-1679.

Sarazin P, Roy X, Favis BD (2004) Controlled preparation and properties of porous poly(L-lactide) obtained from a co-continuous blend of two biodegradable polymers. Biomaterials 25, 5965-5978.

Sofia S, McCarthy MB, Gronowicz G, Kaplan DL (2001) Functionalized silk-based biomaterials for bone formation. J. Biomed. Mater Res 54, 139-148.

Soliman S, Sant S, Nichol JW, Khabiry M, Traversa E, Khademhosseini A (2011) Controlling the porosity of fibrous scaffolds by modulating the fiber diameter and packing density. J. Biomed. Mater Res. Part A 96A, 566?574.

Virgilio N, Desjardins P, Pépin MF, L'Espérance G, Favis BD (2005) High contrast imaging of interphases in ternary polymer blends using focused ion beam preparation and atomic force microscopy. Macromolecules 38, 2368.

Virgilio N, Desjardins P, L'Espérance G, Favis BD (2009) In situ measure of interfacial tensions in ternary and quaternary immiscible polymer blends demonstrating partial wetting. Macromolecules 42, 7518e29.

Virgilio N, Marc-Auréle C, Favis BD (2009) Novel self-assembling close-packed droplet array at the interface in ternary polymer blends. Macromolecules 42, 3405e16.

Virgilio N, Sarazin P, Favis BD (2010) Towards ultraporous poly(L-lactide) scaffolds from quaternary immiscible polymer blends. Biomaterials 31, 5719-5728. 